\title{
Highlighting the advantages and benefits of cementless total knee arthroplasty (Review)
}

\author{
BOGDAN UIVARASEANU ${ }^{1}$, COSMIN MIHAI VESA $^{2}$, DELIA MIRELA TIT $^{3}$, OCTAVIAN MAGHIAR $^{1 *}$, \\ TEODOR ANDREI MAGHIAR ${ }^{1 *}$, CALIN HOZAN $^{1 *}$, AURELIA CRISTINA NECHIFOR $^{4}$, TAPAN BEHL $^{5}$, \\ FELICIA LIANA ANDRONIE-CIOARA ${ }^{6}$, JENEL MARIAN PATRASCU $^{7}$ and SIMONA BUNGAU ${ }^{3}$ \\ Departments of ${ }^{1}$ Surgical Disciplines and ${ }^{2}$ Preclinical Disciplines, Faculty of Medicine and Pharmacy, \\ University of Oradea, 410081 Oradea; ${ }^{3}$ Department of Pharmacy, Faculty of Medicine and Pharmacy, \\ University of Oradea, 410028 Oradea; ${ }^{4}$ Analytical Chemistry and Environmental Engineering Department, \\ Polytechnic University of Bucharest, 011061 Bucharest, Romania; ${ }^{5}$ Department of Pharmacology, \\ Chitkara College of Pharmacy, Chitkara University, Chandigarh, Punjab 140401, India; ${ }^{6}$ Department of \\ Psycho-Neuroscience and Recovery, Faculty of Medicine and Pharmacy, University of Oradea, \\ 410081 Oradea; ${ }^{7}$ Department of Orthopedics, Urology and Medical Imaging, Faculty of Medicine, \\ 'Victor Babes' University of Medicine and Pharmacy, 300041 Timisoara, Romania
}

Received September 3, 2021; Accepted October 5, 2021

DOI: $10.3892 /$ etm.2021.10980

\begin{abstract}
In the field of orthopedic surgery, cemented total knee arthroplasty (TKA) is considered to be one of the gold standards. However, there are categories of patients (i.e., obese and morbidly obese patients, younger than 65 years old) among whom cemented TKA has however a high failure rate. Moreover, the frequency of using uncemented TKA is increasing due to the potential benefits of long-term biological fixation, being an innovative field that addresses a new generation orthopedic surgical treatment which is more suitable for young patients who have good bone quality (good to very good, in terms of density). The survival rates and functional results of the latest generation of cementless TKAs may be similar to functional results and survival rates of cemented prosthesis. In conclusion, this review-type article can be considered a powerful database, extremely informative, dense, and focused
\end{abstract}

Correspondence to: Professor Delia Mirela Tit, Department of Pharmacy, Faculty of Medicine and Pharmacy, University of Oradea, 29 N. Jiga Street, 410028 Oradea, Romania

E-mail:mirela_tit@yahoo.com

Dr Felicia Liana Andronie-Cioara, Department of PsychoNeuroscience and Recovery, Faculty of Medicine and Pharmacy, University of Oradea, 10 1st Decembrie Street, 410081 Oradea, Romania

E-mail: felicia_cioara@yahoo.com

${ }^{*}$ Contributed equally

Key words: uncemented knee arthroplasty, total knee arthroplasty, knee osteoarthritis, gonarthrosis, orthopedic surgery, prosthesis on the topic mentioned above, in the interest of all medical professionals and all interested individuals.

\section{Contents}

1. Introduction

2. Bibliography selection methodology

3. Cementless knee arthroplasty

4. Cementless vs. cemented knee arthroplasty

5. Clinical/therapeutic dilemmas, future directions, and conclusions

\section{Introduction}

The most frequently used procedure in the management of end-stage knee osteoarthritis (OA) is total knee arthroplasty (TKA), that can also be mentioned as relevant for other various underlying manifestations [dysplasia, inflammatory arthritis, malignancy and fracture (deformity and/or post-traumatic OA)] $(1,2)$. With the increased request for TKA operations, so does the variety of the individuals choosing to have the surgical procedure. Previously, TKA was considered a surgical operation for elderly people affected by end-stage arthritis causing persistent knee pain. In recent decades, the number of younger patients and a patient population with greater complexity (from the medical point of view) that undergo TKA has increased considerably $(3,4)$. After the operation, compared to older adults, a critically increased number of younger patients report lower contentment with their functional potential $(5,6)$.

Uncemented knee implants have lately gained further attention due to advanced design processes and manufacturing technologies such as the implementation of an additional coating layer that improves biological adherence and strengthening, as 
well as implant component pattern (7-10). The Miller-Galante II knee, that has remedied the increased incidence of previously observed patellofemoral complications, through improving the implant structure, can be considered an example $(11,12)$. Another reason for the enhanced attention towards cementless fixation is represented by the age of patients subjected to TKA. Younger patients ( $<65$ years), having increased activity levels after the surgery, as well as a greater life expectancy, that need firm, long-lasting fixation techniques increasingly undergo TKA. As the cement mantle does not have remodeling properties such as biologically osseo integrated parts, it is subjected to increased pressure having as a result a greater incidence of aseptic loosening $(10,13)$.

This review refers to a more special topic, namely cementless knee arthroplasty, in terms of the advantages and benefits it offers. This topic was chosen because it is an innovative field that addresses a new generation orthopedic surgical treatment which is more suitable for young patients with bone quality (good to very good, in terms of density). The novelty/special character of this research consists precisely in the exhaustive approach of the numerous aspects related to the cementless TKA.

\section{Bibliography selection methodology}

In order to carry out this review study, the most important databases were researched, of which the richest and most important was PubMed. The search was performed using the key words mentioned at the beginning of the paper, and the articles found were selected based on clear and well-established criteria (English language known by the authors, relevance of the topic addressed, informative nature, scope/novelty of information).

\section{Cementless knee arthroplasty}

Surgical aspects. Following anesthesia, the incision is made on the front part of the knee, to make possible access to the joint. The cutting guide, which is a positioning instrument, is used to perform correct angle bone cutting, preserving the functionality of the joint. Afterwards, the articulating area of the patella is eliminated. The prosthesis elements are pressed to fit on the previously prepared bone surfaces. A metal tray is fixed on the tibia with screws. The screws are kept in place being necessary to fix the implant until enough bone ingrowth develops. To the metal tray is added a plastic spacer that hinders the friction of prosthesis parts. A patellar element is fixed in the rear of the patella, and then the soft tissues are put back into place and sewn. Staples are used to close the skin incision. Obtaining similar flexion and extension joint gaps represents the basis of the articular stability and mobility of the implant. The rotation of the femoral element, that can be attained by ligament balance or bone landmarks, is a key element for this similarity.

There are three bone landmarks that may be used. The trans-epicondylar axis (TEA) represents the link between the groove and the external epicondyle, just under the internal epicondyle. Precisely identifying the TEA is quite difficult, as revealed by many studies (14-16). Using this method indicates an error $>5^{\circ}$ in $56 \%$ of cases (14). The middle of the intercondylar notch is connected to the lowest point in the femoral trochlea at the posterior cruciate ligament (PCL) insertion point by the Whiteside line. It is easily detectable, being perpendicular to the TEA. Bone irregularities modify this line, leading to external rotation flaw in trochlear dysplasia or varus knee osteoarthritis (OA) (15). The posterior condylar axis internally rotated with $3-4^{\circ}$ related to TEA in the normal knee, while a $3-4^{\circ}$ external rotation related to the posterior condyle ensures proper rotational alignment. In valgus knee OA, a confusing hypoplasia of the external condyle is present. On the contrary, in varus knee, anterior cruciate ligament (ACL) deficiency determines the erosion of the medial femoral condyle rear section. To conclude, an exact rotation is achievable in $34 \%$ cases with TEA, in $62 \%$ of cases with posterior condylar axis and in $26 \%$ of cases with Whiteside line (16). The gap balancing method is rather distinct from bone structure and the possible changes. This method implies performing ligamentous release prior to bony resections until an adjustable deformity is obtained. Removing osteophytes is recommendable in order to release tension on the ligaments. The procedure begins with the tibial cut, tensioning the knee in flexion, the tibial cut being parallel to the TEA, that can be used to adjust the alignment. Distal femur cutting is carried out so that the same extension gap is generated (17). Based on these facts, it is considered that the non-cemented prosthesis has to be based on a gap balancing method, reducing stress on uncemented elements, that may trigger micro-movements and changes in the osteointegrative potential, especially at the tibial level (18).

Implant coating material. Among several bioactive coatings that can be used, hydroxyapatite (HA) is applied on the metal substrate of the cementless TKA to improve fixation and transform fibrous tissue into bone $(19,20)$. HA has been demonstrated to lower the tibial component micromotion and improve femoral and tibial element fixation (21). Cross and Parish presented in 2005 the results of a study carried out on 1,000 patients with HA-coated cementless TKA with a 9 -year survey (22). The final evaluation revealed $0.5 \%$ revision rate for aseptic loosening and the 10-year survivorship of the prosthesis of $99.14 \%$ [95\% confidence interval (CI) 92.5 to 99.8 ] (22). A study by Epinette and Manley demonstrated 98.14\% survivorship after an 11.2-year survey of a group of patients (146 primary TKA) with HA-coated cementless prosthesis, considering mechanical failure as end-point (23). The meta-analysis of 926 arthroplasties carried out by Voigt and Mosier revealed that HA-coated devices ensure increased durability compared to other forms of fixation or cemented TKA (24).

A new biomaterial made of tantalum is trabecular metal (Zimmer Biomet) having mechanical characteristics and porosity similar to original trabecular bone (25). Predictable ingrowth of a trabecular metal implant in the surfaces (26) and bone mineral density (BMD) preservation were indicated after ingrowth (27). Increased ratio of early failure for a trabecular metal monoblock tibia was presented by several studies $(28,29)$ including the study of Meneghini and de Beaubien (28), while other studies $(30,31)$ showed positive results, as those of the randomized clinical trial (RCT) performed by Pulido et al on 389 patients monitored for at least 2 years ( 2 to 9 with a mean 5 years) or until death; 128 patients had highly porous metal 
cemented tibia, revealing great bone ingrowth given by the trabecular metal (32).

Uncemented implants have been gradually improved with additional newer concepts. Among these is BIOFOAM (Microport Orthopedics, Inc.) one of the many titanium foams produced by several manufacturers. The foams can be produced with variable porosity and strength of the elements as to be consistent with the bone (up to $80 \%$ porosity) (33). Additional products using electron beam melting (EBM) also known as '3D implants' are other complete orthopaedic implants. This method provides porous metal implant accurate execution. Being able to change porosity and density, these metal parts offer the possibility of improving their biocompatibility. Due to the fact that this method is at the beginning stage, no clinical results are available to date (34).

Benefits. The benefits of cementless implant fixation in knee arthroplasty include: better component fixation directly integrating the bone with the implant covering, genuine bone stock preservation, better surgical efficacy and reduced systemic side effects connected with debris wear and cement impaction $(7,35)$. Cementless implants reduce the incidence of radiological radiolucent lines (RLL) that help increase implant lifespan and decrease the control rates compared to cemented implants for TKA and unicompartmental knee arthroplasty (UKA) (36). These benefits have determined the emergence of high-volume centers selecting cementless over cemented UKA with good outcome reported by patients and over $97 \%$ implant lifespan for at least 10 years of study. The improvement of materials and implant models have determined for TKA a similar evolution with UKA, in the last decade (37).

New improvements in implant models, materials and surgical techniques have enhanced the implant survival rates in contrast to earlier models, reaching a survival rate of $96-100 \%$ after $10-15$ years $(38,39)$. For cemented as well as cementless procedures, UKA has demonstrated better functional results as compared to TKA (40). Conservation of bone stock, maintenance of natural kinematics of the knee, decreased blood loss, preservation of soft tissue anatomy as well as decreased surgical time are among the advantages of UKA (41). There is a significant amount of studies sustaining the mobile bearing unicompartmental implant that was demonstrated to have extremely good functional results with cementless fixation $(42,43)$.

Reviewing the results of several prospective cohort studies on 2,218 cementless UKA, van der List et al presented the component survival at 5,10 and 15 years to be 96.4, 92.9 and $89.3 \%$, respectively. For 10,309 cementless TKAs, the registered outcomes were 97.7, 95.4 and 93\%, respectively (44). However, in spite of the tendency to register data, there are obvious differences between the registered data and the increased implant survival indicated in studies on large groups. However, there is a general favorable tendency of increased implant survival in cementless UKA compared to the cemented alternatives (44-47).

The possibility to obtain durable biological attachment of the implant to the bone, preserving bone stock and preventing cement debris, are theoretically considered advantages of cementless TKA. Loosening may only occur due to lysis or sepsis, being improbable to happen after osseointegration. As a greater number of young and active subjects prefer TKA, a more physiological connection is required (35).

To favor bone formation, cementless implants require roughened or porous surfaces, mesenchymal cells and osteoblasts moving towards the implant (48). The micro-mobility is reduced by the porous surface that ensures a mechanical interlock. Long-term fixation is induced by the initial stability achieved during the operation, being important as micromotion endangers the osseointegration process (49).

\section{Cementless vs. cemented knee arthroplasty}

Cementless prostheses provide advantages such as biological fixation without fragmentation and conservation of the bone stock. Biological fixation of bone prosthesis is desirable due to the lower average age of the patients that undergo arthroplasty (50). Registered data from the UK (51), Sweden (52), Australia (53) and New Zealand (54) have revealed increased use of cemented fixation compared to non-cemented fixation and decreased failure rates. Improved functional results and lower revision rates for cemented TKAs were also presented by several trials $(55,56)$.

Non-cemented fixation is associated with greater damage probability (as the cementless metal-backed element has a thin polyethylene layer) as well as a greater incidence of patellofemoral complications. Combining the evidence from registered data with the results of prospective randomized studies and meta-analyses, Ranawat et al sustain the advantage of cemented fixation in TKAs (57). A meta-analysis conducted by Pijls et al revealed the highest migration for uncoated Interax elements as well as the greatest revision rate for aseptic loosening (58).

Different types of fixations (cemented, uncemented porous HA and uncemented porous fixation) were compared in a study conducted by Carlsson et al who concluded that tibial element cementing provides stabile bone-implant contact for a period of 5 years in comparison with uncemented fixation. Augmenting a porous surface using HA could imply decreased mobility of the implant and bone in the first year after the operation, a fact obvious when uncemented elements are used (59).

Recently, Mont et al identified and reviewed 37 research studies (2,940 patients and 3,568 TKAs) that compared cemented vs. uncemented TKA. A fixed-effect meta-analysis of cemented and cementless TKA as well as cementless TKA with or without screw fixation was used to compare implant survivorship. Implant survivorship of cementless TKA was similar to cemented TKA [odds ratio (OR), 1.1; 95\% confidence interval (CI), 0.62-2.00]. Cementless TKA had an average survivorship at 10 years of $95.6 \%$ while cemented TKA had an average survivorship of $95.3 \%$. Implant survival rate at 20-year revision decreased to 76 and $71 \%$, respectively. There was no difference detected in fixation with or without screws (OR, 1.1; 95\% CI, 0.16-7.5). Cementless TKA implant survival was similar to that of cemented TKAs, the current gold standard (60)

The efficiency of hybrid TKA implants with cementless femoral and cemented tibial elements was evaluated by Lass et al in comparison with titanium-coated cemented implants. A control evaluation of 120 TKAs, 60 hybrid 
cemented and 60 cementless TKAs was conducted by the authors. Clinical and radiographic results as well as the survival rate of the implant at a minimum observation for 5 years were analyzed. The 5-year evaluation was accomplished by 90 patients that were subjected to TKA. Both groups recorded considerably raised Knee Society Scores $(\mathrm{P}<0.001)$. Two patients in both groups needed revision because of aseptic tibial element loosening, the implant survivorship being $96 \%$. Considerably reduced radiolucent lines around the tibial baseplate were detected by radiographs in the cementless group $(n=12)$ compared with the cemented group $(n=26)(P=0.009)$. Taking into account postoperative complications, as well as clinical and functional outcome at a 6-year mean evaluation, there was no considerable discrepancy between cementless and hybrid cemented tibial elements in TKA. Considerably reduced number of radiolucent lines in the cementless group indicated initial stability and the increased fixation durability of TKA (36).

A randomized clinical trial performed on 81 subjects with primary TKA before the age of 70 was conducted by Beaupré et al. The patients were randomly subjected to cemented tibial fixation or cementless tibial fixation. They were evaluated before operation, then at 6 months, 1 year and 5 years after the operation. The 5-year evaluation was completed by 70 subjects (86\%). The RAND-36 and WOMAC scores revealed insignificantly increased pain reported at 6 months in the HA group; 1 year after the operation there were no differences. There were also no differences in radiographic results, complications or function. During the clinical trial, no revision of the tibial prosthesis was necessary. There was no discrepancy between cemented tibial fixation and cementless tibial fixation with HA at 5 years after the operation concerning postoperative complications, health-related quality of life, radiographic, function or self-reported pain (61).

In a study conducted by Bercovy et al on 291 patients, for the ROCC Rotating Platform total knee replacement, the results of 164 cemented elements and 157 HA-coated tibial elements were comparatively analyzed. The average revision was at 7.6 years (range, 5.2-11.0 years). Two revisions were performed for loosening: for cemented tibial element and for an HA-coated tibial element. The HA-coated femoral elements presented no radiolucent lines at the radiological examination. Radiolucent lines were detected at three months after the operation for three HA-coated tibial elements but disappeared after using protective weight-bearing for three more months. In the case of the HA-coated elements, the time for operation was reduced $(\mathrm{P}<0.006)$, the tibial interface radiological evaluation being more stable $(\mathrm{P}<0.01)$. The survivorship at 9 years was $99.1 \%$ for both groups of patients. Similar results were revealed by the findings for cemented elements and HA-coated elements comparable with the results of studies on porous-coated knee replacements or cemented replacements pointing out HA fixation as a good option for initial total knee replacement (62).

A group of 100 TKA subjects randomly distributed to cementless and cemented fixation groups were registered in a study conducted by Fricka et al. The Oxford scores, the KSS functional scores, self-reported satisfaction, better function and reduced pain at 2 years were comparable but increased KSS clinical scores (96.4 vs. 92.3, $\mathrm{P}=0.03$ ) were observed in the cemented group. The cementless knee presented more radiolucencies $(\mathrm{P}<0.001)$. One revision was performed for instability in the cementless group and one for infection in the cemented group. A similar survival rate was indicated for cemented TKA and cementless TKA (revision for any reason as the endpoint) at this interval (63).

Comparable results concerning clinical results, migration and survivorship were revealed by Gao et al. The authors used radiostereometric analysis in an RCT performed on 41 young subjects ( $<60$ years) that underwent TKAs for knee replacement (NexGen, Zimmer Biomet): 19 patients that had hybrid fixated implants and 22 patients having totally cemented implants (64).

Another study carried out by Duffy et al evaluated 55 cementless TKAs compared with 51 cemented TKAs with a 10 -year surveillance. Functional knee and pain scores increased in the first group from 50 and 33 before the operation to 60 and 93 after operation. After 10 years from operation, the survivorship was $72 \%$ and the revision rate for osteolysis or femoral or tibial aseptic loosening was $18.1 \%$ (65).

A systematic review performed by Nakama et al revealed decreased dislocation of the cemented tibial element compared to cementless fixation, in studies performed on patients with rheumatoid arthritis and osteoarthritis that had initial total knee prosthesis, after a surveillance of 2 years; increased risk of aseptic loosening after operation was demonstrated in case of cemented fixation compared to cementless fixation (66).

For subjects $>65$ years, HA-coated tibial implant was found to offer better durability compared to other tibial fixations, a fact revealed by a meta-analysis and systematic review by Voigt and Mosier (24).

The clinical efficacy as well as the reliability of cemented and cementless fixations in primary TKA have been comparatively evaluated in many reviews. The meta-analysis and systematic review performed by Wang et al targeted to use highest-level available evidence to reveal the clinical reliability and functional results of cementless elements compared with the same items for standard cemented elements in young subjects that underwent primary TKA. This meta-analysis was the first research to evaluate cementless compared to cemented TKA in young subjects. The study found cementless fixation as having a better outcome in functional recovery after operation in cases of primary TKA in these patients. RCTs comprising increased quality evidence bases for further use of cementless fixation, providing clinical basis to improve cementless prosthesis were the only ones taken into account for the study (67).

A systematic review of RCTs and a meta-analysis comparatively evaluating the results of cementless and cemented fixation in primary TKA were carried out by Prasad et al. The revision rate was the first evaluated result followed by postoperative functional result scores. Cemented fixation use was found to be similar to cementless fixation use in TKA. The performed research did not reveal a considerable difference in post-operation results, comprising knee function and revision rate of any cause up to 16.6 years. Extended surveillance was recommended based on some evidence, as potential aseptic loosening was revealed in the case of some cementless prosthesis. Because of biological fixation, high post-operation immediate pain, for some patients, was reported with cementless prosthesis (68). A study of 778 subjects under 55 years 
of age with a mean surveillance of 14 years, performed by Gioe et al revealed superior survivorship in the group with cemented TKA. The significance of this result was more illustrative for the cemented group, as a higher number of cemented prosthesis was studied (738 vs. 40 cementless) (69). A retrospective study by Mont $e t$ al revealed no clinical discrepancies in the cemented and cementless groups of young subjects at an average surveillance of 7 years. Being more physically active, the subjects under 65 years of age had greater expectations and exerted greater pressure on the implants (70).

Encouraging long-term outcomes of different cementless devices in comparison with the results of cemented TKA were revealed by many studies $(71,72)$. For 73 Anatomic Graduated Component (AGC, Biomet, Inc.) knees a 20-year survival rate was demonstrated, with 10 year minimal revision and no subject lost, in a study performed by Ritter and Meneghini (73). The study revealed survival rates of $100 \%$ for the cementless femoral elements and survival rates of $96.8 \%$ for the cementless tibial elements when patellar failures were not taken into account (65). A series of 300 consecutive knees arthroplasties were studied by Hofmann et al for 238 patients with a 12-year average evaluation following cementless TKA. In 141 subjects, 176 knees arthroplasties were available for evaluation. Implant survival was $93.4 \%$ (95\% CI 90.1-96.7), at the final evaluation, regardless of polyethylene liner changes and infection. The patellar element survival rate, in the mentioned series was $95.1 \%$ (95\% CI 92.3-98.0) (74).

A rotating platform device was used for treating 169 subjects as reported by Buechel. At 10 and 18 years, the survival rate was $98.3 \%$, with the ending point of evaluation, and $99.4 \%$ survival rate at final evaluation of the cementless patellar elements (71). A total of 255 cementless cruciate-retaining TKAs with unresurfaced patellae were investigated by Whiteside. After surveillance for 15 to 18 years, exceptional Knee Society Scores were reported at final evaluation (75). A prospective research of 76 cementless TKAs performed on 54 patients was conducted by Watanabe et al. The authors reporting a $100 \%$ implant survival rate at 10 years and a 96.7 survival rate at 13 years (76).

\section{Clinical/therapeutic dilemmas, future directions, and conclusions}

The survival rates and functional results of the latest generation of cementless TKAs may be similar to the functional results and survival rates of cemented prosthesis. The novel variants of implants with HA bioactive coatings or of highly porous metals ensure better fixation than other implants as demonstrated by radio-stereographic studies. Due to these technologies, using press-fit stems has become a better option for TKAs for young patients. In order to decide whether to recommend it for large-scale use in TKA, detailed cost analyses are needed. Long-term fixation and biologically active osseointegration are ensured by cementless fixation in TKA. In the first cementless TKA models, failure was observed especially of the tibial and patellar elements. The development of early models, especially in porous metal technology and enhanced biomechanical stability, made cementless TKA important in the last two decades. Compared to the cemented variant, the operation method of cementless TKA is more demanding, emphasizing indications, component alignment as well as accurate resection. Novel cementless TKA models have ensured good early- and mid-term survival rates similar to cemented TKA. Further long-term research is required to look into this issue. Despite the higher costs of cementless implant models, clear evidence shows the lower total cost of cementless elements due to reduced operation time and cement-associated supply costs. There is an increasing interest in cementless implants as the patients requiring TKA are younger and more active. Cementless implant models have been improved in regards to sizing, the metal used and surface adherence. Cemented and cementless elements have been improved with the new results in polyethylene and kinematic topographies. Bone preparation accuracy is possible with an inprovement in instrumentation. Initial implant stability has gained importance.

The acquired information makes way for better results of cementless TKA, needing further research to confirm it. Reliable scientific data sustain the cemented TKA as a reference standard. Cementless TKA is more and more attractive due to the preferences of the patients that require TKA modifications. To assess the correct influence of cementless TKA, further research is required. In the case of subjects with end-stage knee OA, cementless TKA represents a possible fixation alternative even though various studies have questioned the durability of these implants compared to the usual standard cemented TKA. Modern cementless TKA is demonstrated by recent research to have similar results with cemented TKA at the same intervals of surveillance. However, in order to identify the differences between these fixation alternatives more prospective studies and randomized trials are necessary to be carried out.

The current literature offers no evidence in sustaining a particular method of fixation. The extensive use of cemented implants is motivated by the clinical experience obtained in time. Therefore, a comparison between standard cemented implants and ingrowth surface cementless fixation is necessary to be performed using randomized clinical trials. The cemented fixation is unlikely to offer proper long-time results because of fixation failure, due to the ever-increasing demand for TKA and the decreasing age of the OA population. The use of cementless TKA has increased and numerous developments have been made. Modern cementless TKA has similar survival rates and functional results as cemented prostheses, a fact demonstrated by short-term recent trials. Yet, further research is necessary to establish and clarify the discrepancy of these two fixation alternatives.

\section{Acknowledgements}

Not applicable.

\section{Funding}

No funding was received.

\section{Availability of data and materials}

Not applicable. 


\section{Authors' contributions}

BU, CMV, DMT, OM, TAM and CH searched and selected articles from scientific databases. ACN, TB, FLAC, JMP and SB made substantial contributions to the conception of the work; also, they drafted the manuscript and were major contributors in writing the manuscript. All authors read and approved the final manuscript to be published. All the authors agreed to be accountable for all aspects of the work in ensuring that questions related to the accuracy or integrity of any part of the work are appropriately investigated and resolved.

\section{Ethics approval and consent to participate}

Not applicable.

\section{Patient consent for publication}

Not applicable.

\section{Competing interests}

The authors declare that they have no competing interests.

\section{References}

1. Jin ZK, Yang Y, Xu CX, Yang B, Lammi MJ, Chang YH, Ling M, Guo $X$ and Wang XQ: Outcomes of total knee arthroplasty in the adult Kashin-Beck disease with severe osteoarthritis. Int Orthop 43: 323-331, 2019.

2. Gao J, Xing D, Dong S and Lin J: The primary total knee arthroplasty: A global analysis. J Orthop Surg Res 15: 190, 2020.

3. George LK, Hu L and Sloan FA: The effects of total knee arthroplasty on physical functioning and health among the under age 65 population. Value Health 17: 605-610, 2014.

4. Keeney JA, Eunice S, Pashos G, Wright RW and Clohisy JC: What is the evidence for total knee arthroplasty in young patients?: A systematic review of the literature. Clin Orthop Relat Res 469: 574-583, 2011.

5. Nam D, Nunley RM and Barrack RL: Patient dissatisfaction following total knee replacement: A growing concern? Bone Joint J 96-B (11 Suppl A): S96-S100, 2014.

6. Parvizi J, Nunley RM, Berend KR, Lombardi AV Jr, Ruh EL, Clohisy JC, Hamilton WG, Della Valle CJ and Barrack RL: High level of residual symptoms in young patients after total knee arthroplasty. Clin Orthop Relat Res 472: 133-137, 2014.

7. Dalury DF: Cementless total knee arthroplasty: Current concepts review. Bone Joint J 98-B: 867-873, 2016.

8. Meneghini RM and Hanssen AD: Cementless fixation in total knee arthroplasty: Past, present, and future. J Knee Surg 21: 307-314, 2008.

9. Prudhon JL and Verdier R: Cemented or cementless total knee arthroplasty? - Comparative results of 200 cases at a minimum follow-up of 11 years. SICOT J 3: 70, 2017.

10. Kurtz S, Ong K, Lau E, Mowat F and Halpern M: Projections of primary and revision hip and knee arthroplasty in the United States from 2005 to 2030. J Bone Joint Surg Am 89: 780-785, 2007.

11. Effenberger H, Berka J, Hilzensauer G, Ramsauer T, Dorn U and Kisslinger E: Miller-Galante total knee arthroplasty: The importance of material and design on the revision rate. Int Orthop 25: 378-381, 2001

12. Berger RA, Rosenberg AG, Barden RM, Sheinkop MB, Jacobs JJ and Galante JO: Long-term followup of the Miller-Galante total knee replacement. Clin Orthop Relat Res: 58-67, 2001.

13. Losina $\mathrm{E}$ and Katz JN: Total knee arthroplasty on the rise in younger patients: Are we sure that past performance will guarantee future success? Arthritis Rheum 64: 339-341, 2012.

14. Yau W, Chiu K and Tang W: How precise is the determination of rotational alignment of the femoral prosthesis in total knee arthroplasty: An in vivo study. J Arthroplasty 22: 1042-1048, 2007.
15. Poilvache PL, Insall JN, Scuderi GR and Font-Rodriguez DE: Rotational landmarks and sizing of the distal femur in total knee arthroplasty. Clin Orthop Relat Res: 35-46, 1996. doi: 10.1097/0 0003086-199610000-00006.

16. Benjamin J: Determining femoral component position using CAS and measured resection. Clin Orthop Relat Res 466: 2745-2750, 2008.

17. Katz MA, Beck TD, Silber JS, Seldes RM and Lotke PA: Determining femoral rotational alignment in total knee arthroplasty: Reliability of techniques. J Arthroplasty 16: 301-305, 2001.

18. Uivaraseanu B, Bungau S, Tit DM, Behl T, Maghiar TA, Maghiar O, Pantis C, Zaha DC and Patrascu JM: Orthopedic surgery approach with uncemented metallic prosthesis in knee osteoarthritis increases the quality of life of young patients. Exp Ther Med 21: 541, 2021.

19. Søballe K, Hansen E, Brockstedt-Rasmussen H and Bünger C: Hydroxyapatite coating converts fibrous tissue to bone around loaded implants. J Bone Joint Surg Br 75: 270-278, 1993.

20. Tai C and Cross M: Five-to 12-year follow-up of a hydroxyapatitecoated, cementless total knee replacement in young, active patients. J Bone Joint Surg Br 88: 1158-1163, 2006.

21. Toksvig-Larsen S, Jorn LP, Ryd L and Lindstrand A: Hydroxyapatite-enhanced tibial prosthetic fixation. Clin Orthop Relat Res: 192-200, 2000. doi: 10.1097/00003086-20000100000018 .

22. Cross $\mathrm{M}$ and Parish E: A hydroxyapatite-coated total knee replacement: Prospective analysis of 1000 patients. J Bone Joint Surg Br 87: 1073-1076, 2005.

23. Epinette JA and Manley M: Hydroxyapatite-coated total knee replacement: Clinical experience at 10 to 15 years. J Bone Joint Surg Br 89: 34-38, 2007.

24. Voigt JD and Mosier M: Hydroxyapatite (HA) coating appears to be of benefit for implant durability of tibial components in primary total knee arthroplasty. Acta Orthop 82: 448-459, 2011.

25. Dunbar MJ, Wilson DAJ, Hennigar A, Amirault J and Gross M: Fixation of a trabecular metal knee arthroplasty component. A prospective randomized study. J Bone Joint Surg Am 91: 1578-1586, 2009.

26. Henricson A, Linder L and Nilsson KG: A trabecular metal tibial component in total knee replacement in patients younger than 60 years: A two-year radiostereophotogrammetric analysis. J Bone Joint Surg Br 90: 1585-1593, 2008.

27. Harrison AK, Gioe TJ, Simonelli C, Tatman PJ and Schoeller MC: Do porous tantalum implants help preserve bone?: Evaluation of tibial bone density surrounding tantalum tibial implants in TKA. Clin Orthop Relat Res 468: 2739-2745, 2010.

28. Meneghini RM and de Beaubien BC: Early failure of cementless porous tantalum monoblock tibial components. J Arthroplasty 28: 1505-1508, 2013

29. Tigani D, Sabbioni G and Raimondi A: Early aseptic loosening of a porous tantalum knee prosthesis. Chir Organi Mov 93: 187-191, 2009.

30. Ghalayini S and McLauchlan G: Early results of a trabecular metal tibial component in total knee replacement. Orthop Proc Bri Editorial Soc Bone Joint Surg 87B: 145, 2005.

31. Minoda Y, Kobayashi A, Iwaki H, Ikebuchi M, Inori F and Takaoka K: Comparison of bone mineral density between porous tantalum and cemented tibial total knee arthroplasty components. J Bone Joint Surg Am 92: 700-706, 2010

32. Pulido L, Abdel MP, Lewallen DG, Stuart MJ, Sanchez-Sotelo J, Hanssen AD and Pagnano MW: The Mark Coventry Award: Trabecular metal tibial components were durable and reliable in primary total knee arthroplasty: A randomized clinical trial. Clin Orthop Relat Res 473: 34-42, 2015

33. Kato K, Ochiai S, Yamamoto A, Daigo Y, Honma K, Matano S and Omori K: Novel multilayer Ti foam with cortical bone strength and cytocompatibility. Acta Biomater 9: 5802-5809, 2013.

34. Murr LE, Gaytan SM, Martinez E, Medina F and Wicker RB: Next generation orthopaedic implants by additive manufacturing using electron beam melting. Int J Biomater 2012: 245727, 2012.

35. Kurtz SM, Lau E, Ong K, Zhao K, Kelly M and Bozic KJ: Future young patient demand for primary and revision joint replacement: National projections from 2010 to 2030. Clin Orthop Relat Res 467: 2606-2612, 2009.

36. Lass R, Kubista B, Holinka J, Pfeiffer M, Schuller S, Stenicka S, Windhager R and Giurea A: Comparison of cementless and hybrid cemented total knee arthroplasty. Orthopedics 36: e420-e427, 2013 
37. Campi S, Pandit H, Hooper G, Snell D, Jenkins C, Dodd CAF, Maxwell R and Murray DW: Ten-year survival and seven-year functional results of cementless Oxford unicompartmental knee replacement: A prospective consecutive series of our first 1000 cases. Knee 25: 1231-1237, 2018.

38. Zhou K, Yu H, Li J, Wang H, Zhou Z and Pei F: No difference in implant survivorship and clinical outcomes between full-cementless and full-cemented fixation in primary total knee arthroplasty: A systematic review and meta-analysis. Int J Surg 53: 312-319, 2018.

39. Salem HS, Tarazi JM, Ehiorobo JO, Marchand KB, Mathew KK Sodhi N and Mont MA: Cementless fixation for total knee arthroplasty in various patient populations: A literature review. J Knee Surg 33: 848-855, 2020.

40. Wilson HA, Middleton R, Abram SGF, Smith S, Alvand A, Jackson WF, Bottomley N, Hopewell S and Price AJ: Patient relevant outcomes of unicompartmental versus total knee replacement: Systematic review and meta-analysis. BMJ 364: 1352, 2019.

41. Liddle AD, Pandit H, Judge A and Murray DW: Patient-reported outcomes after total and unicompartmental knee arthroplasty: A study of 14076 matched patients from the National Joint Registry for England and Wales. Bone Joint J 97-B: 793-801, 2015.

42. Hasan S, van Hamersveld KT, Marang-van de Mheen PJ, Kaptein BL, Nelissen RG and Toksvig-Larsen S: Migration of a novel 3D-printed cementless versus a cemented total knee arthroplasty: Two-year results of a randomized controlled trial using radiostereometric analysis. Bone Joint J 102: 1016-1024, 2020.

43. Chen $\mathrm{C}$ and Li R: Cementless versus cemented total knee arthroplasty in young patients: A meta-analysis of randomized controlled trials. J Orthop Surg Res 14: 262, 2019.

44. van der List JP, Sheng DL, Kleeblad LJ, Chawla H and Pearle AD: Outcomes of cementless unicompartmental and tota knee arthroplasty: A systematic review. Knee 24: 497-507, 2017.

45. Pandit H, Hamilton T, Jenkins C, Mellon S, Dodd C and Murray D: The clinical outcome of minimally invasive Phase 3 Oxford unicompartmental knee arthroplasty: A 15-year follow-up of 1000 UKAs. Bone Joint J 97-B: 1493-1499, 2015.

46. Baker P, Jameson S, Critchley R, Reed M, Gregg P and Deehan D: Center and surgeon volume influence the revision rate following unicondylar knee replacement: An analysis of 23,400 medial cemented unicondylar knee replacements. J Bone Joint Surg Am 95: 702-709, 2013.

47. Badawy M,Espehaug B, Indrekvam K, Havelin LI and Furnes O: Higher revision risk for unicompartmental knee arthroplasty in low-volume hospitals: Data from 5,791 cases in the Norwegian Arthroplasty Register. Acta Orthop 85: 342-347, 2014.

48. Bobyn JD, Pilliar RM, Cameron HU and Weatherly GC: The optimum pore size for the fixation of porous-surfaced metal implants by the ingrowth of bone. Clin Orthop Relat Res: 263-270, 1980.

49. Cameron HU, Pilliar RM and Macnab I: The effect of movement on the bonding of porous metal to bone. J Biomed Mater Res 7 301-311, 1973.

50. Ponziani L, Di Caprio F and Meringolo R: Cementless knee arthroplasty. Acta Biomed 88: 11-18, 2017.

51. Commitee NS: National Joint Registry for England and Wales: 8th annual report. Hemel Hempstead, UK, 2011.

52. Sundberg M, Lidgren L, Dahl A and Robertson O: The Swedish knee arthroplasty register-Annual report, 2011.

53. Australian Orthopaedic Association National Joint Replacement Registry. Annual Report 2009, 2011

54. Rothwell A, Hobbs T and Frampton C: New Zealand orthopaedic association: New Zealand Joint Registry: Twelve year report January 1999 to December 2010, 2011.

55. Baker PN, Khaw FM, Kirk LM, Esler CN and Gregg PJ: A randomised controlled trial of cemented versus cementless press-fit condylar total knee replacement: 15-year survival analysis. J Bone Joint Surg Br 89: 1608-1614, 2007.

56. van Hamersveld KT, Marang-van de Mheen PJ, Tsonaka R, Valstar ER and Toksvig-Larsen S: Fixation and clinical outcome of uncemented peri-apatite-coated versus cemented total knee arthroplasty: Five-year follow-up of a randomised controlled trial using radiostereometric analysis (RSA). Bone Joint J 99-B: 1467-1476, 2017.

57. Ranawat CS, Meftah M, Windsor EN and Ranawat AS Cementless fixation in total knee arthroplasty: Down the boulevard of broken dreams-affirms. J Bone Joint Surg Br 94 (11 Suppl A): S82-S84, 2012.
58. Pijls BG, Nieuwenhuijse MJ, Schoones JW, Middeldorp S, Valstar ER and Nelissen RG: RSA prediction of high failure rate for the uncoated Interax TKA confirmed by meta-analysis. Acta Orthop 83: 142-147, 2012

59. Carlsson $\AA$, Björkman A, Besjakov J and Önsten I: Cemented tibial component fixation performs better than cementless fixation: A randomized radiostereometric study comparing porous-coated, hydroxyapatite-coated and cemented tibial components over 5 years. Acta Orthop 76: 362-369, 2005.

60. Mont MA, Pivec R, Issa K, Kapadia BH, Maheshwari A and Harwin SF: Long-term implant survivorship of cementless total knee arthroplasty: A systematic review of the literature and meta-analysis. J Knee Surg 27: 369-376, 2014.

61. Beaupré LA, Al-Yamani M, Huckell JR and Johnston DW: Hydroxyapatite-coated tibial implants compared with cemented tibial fixation in primary total knee arthroplasty: A randomized trial of outcomes at five years. J Bone Joint Surg Am 89: 2204-2211, 2007.

62. Bercovy M, Beldame J, Lefebvre B and Duron A: A prospective clinical and radiological study comparing hydroxyapatite-coated with cemented tibial components in total knee replacement. J Bone Joint Surg Br 94: 497-503, 2012.

63. Fricka KB, Sritulanondha S and McAsey CJ: To Cement or Not? Two-Year Results of a Prospective, Randomized Study Comparing Cemented Vs. Cementless Total Knee Arthroplasty (TKA). J Arthroplasty 30 (9 Suppl): 55-58, 2015.

64. Gao F, Henricson A and Nilsson KG: Cemented versus uncemented fixation of the femoral component of the NexGen CR total knee replacement in patients younger than 60 years: A prospective randomised controlled RSA study. Knee 16: 200-206, 2009.

65. Duffy GP, Berry DJ and Rand JA: Cement versus cementless fixation in total knee arthroplasty. Clin Orthop Relat Res: 66-72, 1998. doi: 10.1097/00003086-199811000-00011.

66. Nakama GY, Peccin MS, Almeida GJ, Lira Neto Ode A, Queiroz AA and Navarro RD: Cemented, cementless or hybrid fixation options in total knee arthroplasty for osteoarthritis and other non-traumatic diseases. Cochrane Database Syst Rev 10: CD006193, 2012

67. Wang K, Sun H, Zhang K, Li S, Wu G, Zhou J and Sun X: Better outcomes are associated with cementless fixation in primary total knee arthroplasty in young patients: A systematic review and meta-analysis of randomized controlled trials. Medicine (Baltimore) 99: e18750, 2020.

68. Prasad AK, Tan JHS, Hanna SA, Dawson-Bowling S and Bedair HS: Cemented vs. cementless fixation in primary total knee arthroplasty: A systematic review and meta-analysis. EFORT Open Rev 5: 793-798, 2020.

69. Gioe TJ, Novak C, Sinner P, Ma W and Mehle S: Knee arthroplasty in the young patient: Survival in a community registry. Clin Orthop Relat Res 464: 83-87, 2007.

70. Mont MA, Lee CW, Sheldon M, Lennon WC and Hungerford DS: Total knee arthroplasty in patients. J Arthroplasty 17: 538-543, 2002.

71. Buechel FF Sr: Long-term followup after mobile-bearing total knee replacement. Clin Orthop Relat Res: 40-50, 2002. doi: 10.1 097/00003086-200211000-00008.

72. Hardeman F, Vandenneucker H, Van Lauwe J and Bellemans J: Cementless total knee arthroplasty with Profix: A 8-to 10-year follow-up study. Knee 13: 419-421, 2006.

73. Ritter MA and Meneghini RM: Twenty-year survivorship of cementless anatomic graduated component total knee arthroplasty. J Arthroplasty 25: 507-513, 2010.

74. Hofmann AA, Evanich JD, Ferguson RP and Camargo MP: Ten-to 14-year clinical followup of the cementless Natural Knee system. Clin Orthop Relat Res: 85-94, 2001. doi: 10.1097/00003 086-200107000-00013.

75. Whiteside LA: Choosing your implant: Cementless, patella sparing and posterior cruciate ligament retaining. J Arthroplasty 20 (4 Suppl 2): S10-S11, 2005.

76. Watanabe H, Akizuki S and Takizawa T: Survival analysis of a cementless, cruciate-retaining total knee arthroplasty. Clinical and radiographic assessment 10 to 13 years after surgery. J Bone Joint Surg Br 86: 824-829, 2004. 